\title{
Subset space logic with arbitrary announcements
}

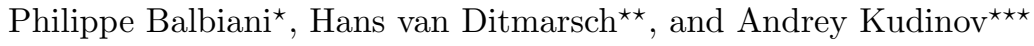 \\ ${ }^{\star}$ Institut de recherche en informatique de Toulouse, CNRS - Université de Toulouse \\ ${ }^{\star \star}$ LORIA, CNRS - Université de Lorraine, France \& IMSc, Chennai, India \\ ${ }^{\star \star \star}$ Institute for Information Transmission Problems RAS \& Higher School of \\ Economics, Moscow, Russia \& Moscow Institute of Physics and Technology, Russia
}

\begin{abstract}
In this paper we introduce public announcements to Subset Space Logic (SSL). In order to do this we have to change the original semantics for SSL a little and consider a weaker version of SSL without the cross axiom. We present an axiomatization, prove completeness and show that this logic is PSPACE-complete. Finally, we add the arbitrary announcement modality which expresses "true after any announcement", prove several semantic results, and show completeness for a Hilbert-style axiomatization of this logic.
\end{abstract}

\section{Introduction}

Subset Space Logic (SSL) was introduced in [6] as logic of knowledge and efforts. The language of SSL includes two modalities $K$ (correspond to knowledge, $K \varphi$ reads as "the agent knows that $\varphi$ is true") and $\square$ (correspond to efforts, $\diamond \varphi=$ $\neg \square \neg \varphi$ reads as " $\varphi$ is true after some efforts"). A formula in this setting evaluates in a pair $(x, U)$, where $x$ is "the actual state of the world" and $U$ is "the epistemic state": the set of states of the world indistinguishable from the real one by the agent. In this context making an effort correspond to shrinking the epistemic state.

Over the years several ways to extend this language were suggested. For example multiple agents were introduced in [13], and the overlap operator in [12]. Another very natural way to extend SSL is with the public announcements operators. The effect of public announcement that $\varphi$ is that the subset space is reduced to all pairs $(x, U)$ that satisfy the formula $\varphi$. In other words, this models some form of external information being provided to the system, that is considered reliable (and thus taken to be true), which results in uncertainty reduction for the knowing agent, but also in uncertainty reduction for the amount of effort needed to make a proposition true or get to know if after that effort: public announcement affects both the $K$ and the $\square$ formulas.

It is intriguing and somewhat of a challenge to distinguish the " $\diamond \varphi$ ", interpreted as " $\varphi$ is true after some effort", from the quantifier ' $\langle!\rangle \varphi$ ', interpreted as " $\varphi$ is true after some announcement". Isn't an announcement also a form of effort? We do not have a conclusive answer to what the difference is, but two suggestions. Firstly, note that the $\varphi$ in $\diamond \varphi$ is interpreted in the same model, not in a changed model, unlike the $\varphi$ in $\langle!\rangle \varphi$ that is interpreted in a model restriction, 
a changed model. Therefore, the $\diamond$ has more the flavor of a conditional logical interpretation (conditional on the agent doing some effort, $\varphi$ is true), unlike the public announcement version. Let " $\psi$ " incorporate the effort; as known, " $\varphi$ is true conditional on $\psi$ " is very different from " $\varphi$ is true after announcement of $\psi$ '. Secondly, we could imagine an application wherein the $\diamond$ in $\diamond \varphi$ represents a form of agency in contrast to $\langle!\rangle \varphi$ that represents the effects of externally driven changes. As known, in public announcement logic there is no clear parallel for agency.

Our main motivation for this logic was to demonstrate that one can fruitfully add a dynamic aspect similar to that in dynamic epistemic logic to a very different logic, and "make it work".

A first attempt to extend SSL with public announcements was by Can Başkent in his master thesis [3]. We think that this semantics for public announcement in SSL is not well-defined; and also other intrinsic problems are not easy to overcome (see Appendix). To address these issues we propose a weaker version of SSL (wSSL) without the cross axiom; and to prove completeness we also modified the semantics somewhat.

We further extended this public announcement SSL with the arbitrary/any announcement operator of [2]. This models what can be known and which further effort still needs to be taken (in the SSL setting) after any announcement, i.e., after any external information has been incorporated.

We should also mention the work of Ågotnes and Wáng [1] where they take a different approach. Instead of adding public announcements operators to SSL they give an alternative semantics for PAL, using subset spaces instead of model updates.

\section{Subset space logic}

\subsection{Syntax and semantics}

Let $\operatorname{Var}$ be a countable set of propositional variables (with typical members denoted $p, q$, etc). The set For of all formulas over Var (with typical members denoted $\varphi, \psi$, etc) is defined by the rule

$$
\varphi::=p|\perp| \neg \varphi|(\varphi \vee \psi)| K \varphi \mid \square \varphi .
$$

It is usual to omit parentheses if this does not lead to any ambiguity. We shall say that a formula $\varphi$ is Boolean iff $\varphi$ contains neither the modal connective $K$, nor the modal connective $\square$. The notion of a subformula is standard. We adopt the standard definitions for the remaining Boolean connectives. As usual, we define $\hat{K} \varphi::=\neg K \neg \varphi$ and $\diamond \varphi::=\neg \square \neg \varphi$. We inductively define the degree of a formula $\varphi$ (denoted $\operatorname{deg}(\varphi))$ as follows: (i) $\operatorname{deg}(p)=0$; (ii) $\operatorname{deg}(\perp)=0$; (iii) $\operatorname{deg}(\neg \varphi)=$ $\operatorname{deg}(\varphi)$; (iv) $\operatorname{deg}(\varphi \vee \psi)=\max (\{\operatorname{deg}(\varphi), \operatorname{deg}(\psi)\}) ;(\mathbf{v}) \operatorname{deg}(K \varphi)=\operatorname{deg}(\square \varphi)=$ $\operatorname{deg}(\varphi)+1$.

Let $|\varphi|$ denote the length of $\varphi$ and $\operatorname{Var}(\varphi)$ be the set variables in $\varphi$. 
Definition 1. A (wSSL-)frame is a structure of the form $\mathcal{F}=(X, S, W)$ where $X$ is a nonempty set of states (denoted $x, y$, etc), $S \subset \mathcal{P}(X)$ is a nonempty set of nonempty subsets of $X$ (denoted $U, V$, etc) and $W$ is a nonempty set of pairs $(x, U)$ such that $x \in X, U \in S$ and $x \in U$. Given a frame $\mathcal{F}=(X, S, W)$, let $\rightarrow_{K}^{\mathcal{F}}$ and $\rightarrow \underset{\square}{\mathcal{F}}$ be the binary relations on $W$ defined as follows: (i) $(x, U) \rightarrow_{K}^{\mathcal{F}}(y, V)$ iff $U=V$; (ii) $(x, U) \rightarrow \underset{\square}{\mathcal{F}}(y, V)$ iff $x=y$ and $U \supseteq V$.

Note that in this definition set $S$ does not play any significant role and can be replaced with $\mathcal{P}(X)$ without any effect on validity.

We show first that

Lemma 1. 1. $\rightarrow_{K}^{\mathcal{F}}$ is an equivalence relation.

2. $\rightarrow{ }_{\square}^{\mathcal{F}}$ is reflexive and transitive.

Definition 2. Given a frame $\mathcal{F}=(X, S, W)$, a valuation on $\mathcal{F}$ is a function $\theta$ assigning to each $p \in$ Var a subset $\theta(p)$ of $X$. We inductively define the satisfaction of a formula $\varphi$ in a frame $\mathcal{F}=(X, S, W)$ with respect to a valuation $\theta$ on $\mathcal{F}$ at $(x, U) \in W$ (denoted $\mathcal{F}, \theta,(x, U) \models \varphi$ ) as follows:

$-\mathcal{F}, \theta,(x, U) \models p$ iff $x \in \theta(p)$;

- all logical connectives are treated as usual;

- $\mathcal{F}, \theta,(x, U) \models K \varphi$ iff $\forall(y, V) \in W\left((x, U) \rightarrow_{K}^{\mathcal{F}}(y, V) \Rightarrow \mathcal{F}, \theta,(y, V) \models \varphi\right)$;

- $\mathcal{F}, \theta,(x, U) \models \square \varphi$ iff $\forall(y, V) \in W((x, U) \rightarrow \underset{\square}{\mathcal{F}}(y, V) \Rightarrow \mathcal{F}, \theta,(y, V) \models \varphi)$.

Remark. If for some $S \subseteq 2^{X}$ we take $W=\{(x, U) \mid x \in X, x \in U \in S\}$ then frame $(X, S, W)$ is equivalent to (validates the same formulas) the classical subset space $(X, S, \theta)$ (see [6]). So these models can be viewed at as a generalization of subset spaces. Consider the cross axiom $(C A=\diamond \hat{K} p \rightarrow \hat{K} \diamond p)$ which is valid in any classical subset space and can be false in a wSSL-model. Indeed consider two sets $V \subset U$ and two points $x, y \in V$ such that $\{(x, V),(x, U),(y, V)\}=W$ and $(y, V) \models p$, then $(x, U) \models \diamond \hat{K} p \wedge \neg \hat{K} \diamond p$.

We shall say that a formula $\varphi$ is universally satisfied in a frame $\mathcal{F}=(X, S, W)$ with respect to a valuation $\theta$ on $\mathcal{F}$ (denoted $\mathcal{F}, \theta=\varphi$ ) iff for all $(x, U) \in W$, $\mathcal{F}, \theta,(x, U) \models \varphi$. A formula $\varphi$ is said to be valid in a frame $\mathcal{F}=(X, S, W)$ (denoted $\mathcal{F}=\varphi$ ) iff for all valuations $\theta$ on $\mathcal{F}, \mathcal{F}, \theta=\varphi$. We shall say that a formula $\varphi$ is valid (denoted $\models \varphi$ ) iff for all frames $\mathcal{F}=(X, S, W), \mathcal{F} \models \varphi$. So, by Lemma 1 and standard arguments we have

Proposition 1. 1. $\models K \varphi \rightarrow \varphi, \models \varphi \rightarrow K \hat{K} \varphi$ and $\models K \varphi \rightarrow K K \varphi$.

2. $\models \square \varphi \rightarrow \varphi$ and $\models \square \varphi \rightarrow \square \square \varphi$.

Proposition 2. If $\varphi$ is a Boolean formula then $\models \varphi \rightarrow \square \varphi$.

\subsection{Axiomatization and completeness}

The axioms of $w S S L$ are all instances of Boolean tautologies plus the following formulas: (i) $K(\varphi \rightarrow \psi) \rightarrow(K \varphi \rightarrow K \psi)$; (ii) $\square(\varphi \rightarrow \psi) \rightarrow(\square \varphi \rightarrow \square \psi)$; (iii) $K \varphi \rightarrow \varphi$; (iv) $\varphi \rightarrow K \hat{K} \varphi$; (v) $K \varphi \rightarrow K K \varphi$; (vi) $\square \varphi \rightarrow \varphi$; (vii) $\square \varphi \rightarrow$ 
$\square \square \varphi$; (viii) if $\varphi$ is a Boolean formula then $\varphi \rightarrow \square \varphi$. The rules of inference of $w S S L$ are: (i) modus ponens (from $\varphi$ and $\varphi \rightarrow \psi$ infer $\psi$ ); (ii) $K$-generalization (from $\varphi$ infer $K \varphi$ ); (iii) $\square$-generalization (from $\varphi$ infer $\square \varphi$ ). A formula $\varphi$ is said to be $w S S L$-provable iff $\varphi$ belongs to the least set of formulas containing all axioms of $w S S L$ and closed with respect to all rules of inference of $w S S L$.

Using induction one can easily prove

Proposition 3. Let $\varphi$ be a formula. If $\varphi$ is $w S S L$-provable then $\models \varphi$.

The following result is expected but more difficult to prove.

Proposition 4. Let $\varphi$ be a formula. If $\models \varphi$ then $\varphi$ is $w S S L$-provable.

We shall say that a set $\Gamma$ of formulas is a $w S S L$-theory iff it satisfies the following conditions: (i) $\Gamma$ contains the set of all $w S S L$-provable formulas; (ii) $\Gamma$ is closed under the rule of inference of modus ponens. Obviously, the least $w S S L$-theory is the set $\operatorname{Pr}(w S S L)$ of all $w S S L$-provable formulas whereas the greatest $w S S L$ theory is the set of all formulas. A $w S S L$-theory $\Gamma$ is said to be consistent iff $\perp \notin \Gamma$. Let us remark that the only inconsistent $w S S L$-theory is the set of all formulas. We shall say that a $w S S L$-theory $\Gamma$ is maximal iff for all formulas $\varphi, \varphi \in \Gamma$, or $\neg \varphi \in \Gamma$. Let $\Gamma$ be a $w S S L$-theory. For all formulas $\varphi$, let $\Gamma+\varphi$ be the set of all formulas $\psi$ such that $\varphi \rightarrow \psi \in \Gamma$. It is a simple matter to check that $\Gamma+\varphi$ is a $w S S L$-theory. Moreover, $\Gamma+\varphi$ is consistent iff $\neg \varphi \notin \Gamma$. The proposition below is a variant of well known Lindenbaum's lemma. See [5, Lemma 4.17] for the proof of a similar result.

Proposition 5. Let $\Gamma$ be a wSSL-theory. If $\Gamma$ is consistent then there exists a maximal consistent $w S S L$-theory $\Delta$ such that $\Gamma \subseteq \Delta$.

Let $\Gamma$ be a $w S S L$-theory. Let: (i) $K \Gamma$ be the set of all formulas $\varphi$ such that $K \varphi \in \Gamma$; (ii) $\square \Gamma$ be the set of all formulas $\varphi$ such that $\square \varphi \in \Gamma$. It is easy to prove that $K \Gamma$ is a $w S S L$-theory and $\square \Gamma$ is a $w S S L$-theory using distribution axioms and $\square$ - and $K$-generalization rules.

Our first task is to define the canonical model of $w S S L$. The canonical model of $w S S L$ is the structure $\mathcal{M}^{c}=\left(S^{c}, R_{K}^{c}, R_{\square}^{c}, \theta^{c}\right)$ defined as follows: (i) $S^{c}$ is the set of all maximal consistent $w S S L$-theories; (ii) $R_{K}^{c}$ is the binary relation on $S^{c}$ defined by $\Gamma R_{K}^{c} \Delta$ iff $K \Gamma \subseteq \Delta$; (iii) $R_{\square}^{c}$ is the binary relation on $S^{c}$ defined by $\Gamma R_{\square}^{c} \Delta$ iff $\square \Gamma \subseteq \Delta$; (iv) $\theta^{c}$ is the function assigning to each $p \in \operatorname{Var}$ the subset $\theta^{c}(p)$ of $S^{c}$ defined by $\Gamma \in \theta^{c}(p)$ iff $p \in \Gamma$. It is worth noting at this point the following:

Lemma 2. 1. $R_{K}^{c}$ is an equivalence relation.

2. $R_{\square}^{c}$ is reflexive and transitive.

Considering $\mathcal{M}^{c}$ as a Kripke model where the modal connectives $K$ and $\square$ are interpreted by means of the binary relations $R_{K}^{c}$ and $R_{\square}^{c}$, the proposition below contains a result that can be proved by induction on $\varphi$. See [5, Lemma 4.21] for the proof of a similar result.

Proposition 6. Let $\varphi$ be a formula. For all $\Gamma \in S^{c}$, we have $\mathcal{M}^{c}, \Gamma \models \varphi$ iff $\varphi \in \Gamma$. 
Let $\Gamma_{0}$ be a maximal consistent $w S S L$-theory. Our second task is to unravel $\mathcal{M}^{c}$ around $\Gamma_{0}$. The unraveling of $\mathcal{M}^{c}$ around $\Gamma_{0}$ is the structure $\mathcal{M}^{u}=$ $\left(S^{u}, R_{K}^{u}, R_{\square}^{u}, \theta^{u}\right)$ defined as follows: (i) $S^{u}$ is the set of all finite sequences $\left(i_{1}, \Gamma_{1}, \ldots, i_{m}, \Gamma_{m}\right)$ such that $m$ is a nonnegative integer, $i_{1}, \ldots, i_{m} \in\{K, \square\}$ and $\Gamma_{1}, \ldots, \Gamma_{m} \in S^{c}$ are such that $\Gamma_{0} R_{i_{1}}^{c} \Gamma_{1}, \ldots, \Gamma_{m-1} R_{i_{m}}^{c} \Gamma_{m}$; (ii) $R_{K}^{u}$ is the binary relation on $S^{u}$ defined by $\left(i_{1}, \Gamma_{1}, \ldots, i_{m}, \Gamma_{m}\right) R_{K}^{u}\left(j_{1}, \Delta_{1}, \ldots, j_{n}, \Delta_{n}\right)$ iff there exists a nonnegative integer $o$ such that $o \leq m, o \leq n,\left(i_{1}, \Gamma_{1}, \ldots, i_{o}, \Gamma_{o}\right)=$ $\left(j_{1}, \Delta_{1}, \ldots, j_{o}, \Delta_{o}\right), i_{o+1}=\ldots=i_{m}=K$ and $j_{o+1}=\ldots=j_{n}=K$; (iii) $R_{\square}^{u}$ is the binary relation on $S^{u}$ defined by $\left(i_{1}, \Gamma_{1}, \ldots, i_{m}, \Gamma_{m}\right) R_{\square}^{u}\left(j_{1}, \Delta_{1}, \ldots, j_{n}, \Delta_{n}\right)$ iff $m \leq n,\left(i_{1}, \Gamma_{1}, \ldots, i_{m}, \Gamma_{m}\right)=\left(j_{1}, \Delta_{1}, \ldots, j_{m}, \Delta_{m}\right)$ and $j_{m+1}=\ldots=j_{n}=\square$; (iv) $\theta^{u}$ is the function assigning to each $p \in \operatorname{Var}$ the subset $\theta^{u}(p)$ of $S^{u}$ defined by $\left(i_{1}, \Gamma_{1}, \ldots, i_{m}, \Gamma_{m}\right) \in \theta^{u}(p)$ iff $p \in \Gamma_{m}$. We adopt the convention that an empty sequence (say, when $m=0$, or $n=0$ above) has value $\Gamma_{0}$. For all $\left(i_{1}, \Gamma_{1}, \ldots, i_{m}, \Gamma_{m}\right) \in S^{u}$, let $\sharp_{\square}\left(i_{1}, \Gamma_{1}, \ldots, i_{m}, \Gamma_{m}\right)=\operatorname{Card}(\{\alpha: \alpha$ is a positive integer such that $\alpha \leq m$ and $\left.\left.i_{\alpha}=\square\right\}\right)$. By Lemma 2, we infer immediately the following.

Lemma 3. 1. $R_{K}^{u}$ is an equivalence.

2. $R_{\square}^{u}$ is reflexive and transitive.

Considering $\mathcal{M}^{u}$ as a Kripke model where the modal connectives $K$ and $\square$ are interpreted by means of the binary relations $R_{K}^{u}$ and $R_{\square}^{u}$, the proposition below contains a result that can be proved by induction on $\varphi$.

Proposition 7. Let $\varphi$ be a formula. For all $\left(i_{1}, \Gamma_{1}, \ldots, i_{m}, \Gamma_{m}\right) \in S^{u}$, we have $\mathcal{M}^{u},\left(i_{1}, \Gamma_{1}, \ldots, i_{m}, \Gamma_{m}\right) \models \varphi$ iff $\mathcal{M}^{c}, \Gamma_{m} \models \varphi$.

Proof. See [5, Lemma 4.52] for the proof that $\mathcal{M}^{c}$ is a bounded morphic image of $\mathcal{M}^{u}$ and [5, Proposition 2.14] for the proof that modal satisfaction is invariant under bounded morphisms.

Let $\equiv^{u}$ be the symmetric and transitive closure of $R_{\square}^{u}$ and $\preceq^{u}$ be the transitive closure of $R_{K}^{u} \circ R_{\square}^{u}$. Obviously, $\equiv^{u}$ is reflexive, symmetrical and transitive and $\preceq^{u}$ is reflexive and transitive. Let $\Gamma \in S^{u}$. The equivalence class modulo $\equiv^{u}$ with $\boldsymbol{\Gamma}$ as its representative is denoted $[\boldsymbol{\Gamma}]_{\equiv u}$. The set of all equivalence classes of $S^{u}$ modulo $\equiv^{u}$ is denoted $S^{u} / \equiv^{u}$. Let us define function $f: S^{u} \rightarrow \mathcal{P}\left(S^{u} / \equiv^{u}\right)$ defined as follows

$$
f(\boldsymbol{\Gamma})=\left\{[\boldsymbol{\Gamma}]_{\equiv^{u}} \mid \boldsymbol{\Gamma} \preceq^{u} \boldsymbol{\Delta}\right\} .
$$

To continue, another technical lemma is necessary.

Lemma 4. Let $\boldsymbol{\Gamma}, \boldsymbol{\Delta} \in S^{u}$.

1. If $f(\boldsymbol{\Gamma})=f(\boldsymbol{\Delta})$ then $\boldsymbol{\Gamma} R_{K}^{u} \boldsymbol{\Delta}$.

2. If $\boldsymbol{\Gamma} \equiv{ }^{u} \boldsymbol{\Delta}$ and $f(\boldsymbol{\Gamma}) \supseteq f(\boldsymbol{\Delta})$ then $\boldsymbol{\Gamma} R_{\square}^{u} \boldsymbol{\Delta}$.

Our third task is to spatialize $\mathcal{M}^{u}$. The spatialization of $\mathcal{M}^{u}$ consists of the frame $\mathcal{F}^{s}=\left(X^{s}, S^{s}, W^{s}\right)$ and the valuation $\theta^{s}$ on $\mathcal{F}^{s}$ defined as follows: (i) $X^{s}=S^{u} /{ }^{u}$; (ii) $S^{s}$ is the range of $f$; (iii) $W^{s}=\left\{\left([\boldsymbol{\Gamma}]_{\equiv u}, f(\boldsymbol{\Gamma})\right) \mid \boldsymbol{\Gamma} \in S^{u}\right\}$; (iv) valuation $\theta^{s}$ is as follows $\theta^{s}(p)=\left\{[\boldsymbol{\Gamma}]_{\equiv u} \mid \boldsymbol{\Gamma} \in \theta^{u}(p)\right\}$. The interesting result is the following 
Proposition 8. Let $\varphi$ be a formula. For all $\boldsymbol{\Gamma} \in S^{u}$, we have $\mathcal{F}^{s}, \theta^{s},\left([\boldsymbol{\Gamma}]_{\equiv}, f(\boldsymbol{\Gamma})\right) \models \varphi$ iff $\mathcal{M}^{u}, \boldsymbol{\Gamma} \models \varphi$.

Now, we can proceed to the

Proof of Proposition 4. Suppose $\varphi$ is not $w S S L$-provable. Hence, $\operatorname{Pr}(w S S L)+\neg \varphi$ is a consistent $w S S L$-theory. Thus, by Proposition 5, there exists a maximal consistent $w S S L$-theory $\Gamma_{0}$ such that $\operatorname{Pr}(w S S L)+\neg \varphi \subseteq \Gamma_{0}$. Obviously, $\varphi \notin$ $\Gamma_{0}$. Therefore, by Proposition $6, \mathcal{M}^{c}, \Gamma_{0} \forall \varphi$. Consequently, by Proposition 7 , $\mathcal{M}^{u}, \Gamma_{0} \not \models \varphi$. Hence, by Proposition $8, \mathcal{F}^{s}, \theta^{s},\left(\left[\Gamma_{0}\right]_{\equiv u}, f\left(\Gamma_{0}\right)\right) \not \models \varphi$. Thus, $\forall \varphi$. $\dashv$

\subsection{Decidability and complexity}

Fix a formula $\varphi$ with $\operatorname{deg}(\varphi)=k$. Let $\varphi^{\star}$ be the conjunction of the following formulas: (i) $\neg \varphi$; (ii) for all $p \in \operatorname{Var}(\varphi),(K \square)^{k}(p \rightarrow \square p)$; (iii) for all $p \in$ $\operatorname{Var}(\varphi),(K \square)^{k}(\neg p \rightarrow \square \neg p)$. In the above formulas, $(K \square)^{k}$ means $K \square$ repeated $k$ times. We first prove a simple lemma.

Lemma 5. The following conditions are equivalent:

1. $\varphi^{\star}$ is satisfied in a Kripke model of the form $\mathcal{M}=\left(S, R_{K}, R_{\square}, \theta\right)$ where $R_{K}$ is reflexive, symmetrical and transitive, $R_{\square}$ is reflexive and transitive and the modal connectives $K$ and $\square$ are interpreted by means of the binary relations $R_{K}$ and $R_{\square}$.

2. $\forall \varphi \varphi$.

Proposition 9. The membership problem in the set of all valid formulas is in PSPACE.

Proof. By Lemmas 5, the membership problem in the set of all valid formulas is reducible to the membership problem in $S 5 \otimes S 4$. Since the membership problem in $S 5 \otimes S 4$ is in $P S P A C E$ [14, Theorem 7], then the membership problem in the set of all valid formulas is in PSPACE.

Let $Q_{1} p_{1} \ldots Q_{n} p_{n} \varphi\left(p_{1}, \ldots, p_{n}\right)$ be a $\mathrm{QBF}$ and consider the new propositional variables $q_{0}, q_{1}, \ldots, q_{n}$. Let $\left[Q_{1} p_{1} \ldots Q_{n} p_{n} \varphi\left(p_{1}, \ldots, p_{n}\right)\right]$ be the conjunction of the following formulas: (i) $q_{0}$; (ii) $K \square\left(q_{i-1} \rightarrow \hat{K} \diamond\left(q_{i} \wedge K p_{i}\right) \wedge \hat{K} \diamond\left(q_{i} \wedge K \neg p_{i}\right)\right)$ for each positive integer $i$ such that $i \leq n$ and $Q_{i}=\forall$; (iii) $K \square\left(q_{i-1} \rightarrow\right.$ $\left.\hat{K} \diamond\left(q_{i} \wedge K p_{i}\right) \vee \hat{K} \diamond\left(q_{i} \wedge K \neg p_{i}\right)\right)$ for each positive integer $i$ such that $i \leq n$ and $Q_{i}=\exists$; (iv) $K\left(q_{n} \rightarrow \varphi\right)$. The next lemma explains the relationship between $\left[Q_{1} p_{1} \ldots Q_{n} p_{n} \varphi\left(p_{1}, \ldots, p_{n}\right)\right]$ and $Q_{1} p_{1} \ldots Q_{n} p_{n} \varphi\left(p_{1}, \ldots, p_{n}\right)$.

Lemma 6. $A Q A=Q_{1} p_{1} \ldots Q_{n} p_{n} \varphi\left(p_{1}, \ldots, p_{n}\right)$ holds iff $[A]$ is satisfied.

Proposition 10. The membership problem in the set of all valid formulas is PSPACE-hard.

Proof. By Lemma 6, the QBF-validity problem is reducible to the membership problem in the set of all valid formulas. Since the QBF-validity problem is $P S P A C E$-hard [16, Theorem 19.1], then the membership problem in the set of all valid formulas is $P S P A C E$-hard. 


\section{Subset space logic with announcements}

\subsection{Syntax and semantics}

We consider an extension $w S S L_{a}$ of $w S S L$ with announcements operators.

The set For ${ }_{a}$ of all formulas with announcements over $\operatorname{Var}$ (with typical members denoted $\varphi, \psi$, etc) is defined by the rule

$$
\varphi::=p|\perp| \neg \varphi|(\varphi \vee \psi)| K \varphi|\square \varphi|[\varphi] \psi
$$

We define $\langle\varphi\rangle \psi::=\neg[\varphi] \neg \psi$.

The definition of the satisfiability of the formula $[\varphi] \psi$ in a frame $\mathcal{F}=$ $(X, S, W)$ with respect to a valuation $\theta$ on $\mathcal{F}$ at $(x, U) \in W$ is defined as follows: $\mathcal{F}, \theta,(x, U) \models[\varphi] \psi$ iff if $\mathcal{F}, \theta,(x, U) \models \varphi$ then $\left(X, S, W_{\mid \varphi}\right), \theta,(x, U) \models \psi$ where $W_{\mid \varphi}=\{(y, V):(y, V) \in W$ is such that $\mathcal{F}, \theta,(y, V) \models \varphi\}$. The following propositions are basic.

Proposition 11. The following formulas are valid: $[\varphi] p \leftrightarrow(\varphi \rightarrow p),[\varphi] \perp \leftrightarrow$ $\neg \varphi,[\varphi] \neg \psi \leftrightarrow(\varphi \rightarrow \neg[\varphi] \psi),[\varphi](\psi \vee \chi) \leftrightarrow([\varphi] \psi \vee[\varphi] \chi),[\varphi] K \psi \leftrightarrow(\varphi \rightarrow K[\varphi] \psi)$, $[\varphi] \square \psi \leftrightarrow(\varphi \rightarrow \square[\varphi] \psi),[\varphi][\psi] \chi \leftrightarrow[\langle\varphi\rangle \psi] \chi$.

Let $t r: F_{0} r_{a} \rightarrow$ For be the standard meaning-preserving translation from For $_{a}$ to For. It can be defined inductively in a standard way using equivalences from Proposition 11. This translation has been considered in several places (cf. [7]).

Proposition 12. For all formulas $\varphi$ in For $_{a}$, there exists a formula $\psi(=\operatorname{tr}(\varphi))$ in For such that $\models \varphi \leftrightarrow \psi$.

\subsection{Axiomatization/completeness}

The axioms of $w S S L_{a}$ are all axioms of $w S S L$ plus all the formulas from Proposition 11. The rules of inference of $w S S L_{a}$ are all rules of inference of $w S S L$ plus the following rule of inference: $[\varphi]$-generalization (from $\psi$ infer $[\varphi] \psi$ ).

For our purpose, the following crucial property of the translation $t r$ can be proved by induction.

Proposition 13. Let $\varphi$ be a formula in $\operatorname{For}_{a} . \operatorname{tr}(\varphi) \leftrightarrow \varphi$ is $w S S L_{a}$-provable. And if $\varphi$ is $w S S L_{a}$-provable then $\models \varphi$.

Referring to Proposition 4, we obtain the

Proposition 14. Let $\varphi$ be a formula in For F $_{\text {. If }}=\varphi$ then $\varphi$ is $w S S L_{a}$-provable.

Proof. Suppose $\varphi$ is not $w S S L_{a}$-provable. Hence, by Proposition $13, \operatorname{tr}(\varphi)$ is not $w S S L_{a}$-provable. Thus, $\operatorname{tr}(\varphi)$ is not $w S S L$-provable. Therefore, by Proposition $4, \not=\operatorname{tr}(\varphi)$. Consequently, by Proposition $12, \forall \neq \varphi$. 


\subsection{Decidability and complexity}

We will following the line of reasoning suggested in [15]. Proof details are omitted.

Proposition 15. The membership problem in the set of all valid formulas is in PSPACE.

Proposition 16. The membership problem in the set of all valid formulas is PSPACE-hard.

Proof. By Proposition 10.

\section{Subset space logic with arbitrary announcements}

\subsection{Syntax and semantics}

We consider an extension $w S S L_{a a}$ of $w S S L_{a}$ wherein we can express what becomes true without explicit reference to announcements realizing that.

The set For ${ }_{a a}$ of all formulas with arbitrary announcements over Var (with typical members denoted $\varphi, \psi$, etc) is defined by the rule

$$
\varphi::=p|\perp| \neg \varphi|(\varphi \vee \psi)| K \varphi|\square \varphi|[\varphi] \psi \mid[!] \varphi .
$$

We define $\langle!\rangle \varphi::=\neg[!] \neg \varphi$. For the definition of the [!]-special rule of inference in section 4.2, we will need formulas of a special form, called admissible forms. Let $\sharp$ be a new propositional variable. The set $A F(\operatorname{Var})$ of all admissible forms over $\operatorname{Var}$ (with typical members denoted $A, B$, etc) is defined by the rule

$$
A::=\sharp|\varphi \rightarrow A| K A \mid \square A .
$$

Note that in each admissible form $A, \sharp$ has a unique occurrence. Given an admissible form $A(\sharp)$ and a formula $\varphi$, let $A(\varphi)$ be the result of the replacement of $\sharp$ in its place in $A$ with $\varphi$. The definition of the satisfiability of the formula [!] $\varphi$ in a frame $\mathcal{F}=(X, S, W)$ with respect to a valuation $\theta$ on $\mathcal{F}$ at $(x, U) \in W$ is defined as follows: $\mathcal{F}, \theta,(x, U) \models[!] \varphi$ iff for all formulas $\psi$ in $F_{\text {For }} a$, if $\mathcal{F}, \theta,(x, U) \models \psi$ then $\left(X, S, W_{\mid \psi}\right), \theta,(x, U) \models \psi$. The following propositions are basic.

Proposition 17. The following formulas are valid: [!] $\rightarrow \varphi,[!] \varphi \rightarrow[!][!] \varphi$, $[!]\langle!\rangle \varphi \rightarrow\langle!\rangle[!] \varphi,\langle!\rangle[!] \varphi \rightarrow[!]\langle!\rangle \varphi$.

The following proposition can be proved similar to Proposition 3.9 in [2].

Proposition 18. $\models K[!] \varphi \rightarrow[!] K \varphi$.

Although for all formulas $\varphi, K[!] \varphi \rightarrow[!] K \varphi$ is valid, there exists formulas $\varphi$ such that $[!] K \varphi \rightarrow K[!] \varphi$ is not valid.

Example 1. For example, one may consider the formula $\varphi=\diamond \hat{K} p$. In the frame $\mathcal{F}=(X, S, W)$ where $X=\{x, y\}, S=\{\{x\},\{x, y\}\}$ and $W=$ $\{(x,\{x\}),(x,\{x, y\}),(y,\{x, y\})\}$, with respect to a valuation $\theta$ on $\mathcal{F}$ such that $\theta(p)=\{x\},(x,\{x, y\})$ does not satisfy $[!] K \varphi \rightarrow K[!] \varphi$. 
Let us show that there exists a formula that is equivalent to no formula in For . $_{\text {. }}$

Example 2. To illustrate the truth of this, take the case of the formula $\varphi=$ $[!](\square \hat{K} \diamond K p \rightarrow \diamond K p)$ and assume that [!]-free formula $\psi$ is equivalent to $\varphi$ and let $q$ is a new variable $q \notin \operatorname{Var}(\psi)$. Consider the frame $\mathcal{F}=(X, S, W)$ where $X=\{x, y\}, S=\{\{x, y\}\}$ and $W=\{(x,\{x, y\}),(y,\{x, y\})\}$, the valuation $\theta$ on $\mathcal{F}$ such that $\theta(p)=\{x\}, \theta(q)=\emptyset$ and $\theta(r)=\emptyset$ for each propositional variable $r \neq p, q$, the frame $\mathcal{F}^{\prime}=\left(X^{\prime}, S^{\prime}, W^{\prime}\right)$ where

$$
\begin{aligned}
X^{\prime} & =\left\{x_{1}^{\prime}, x_{2}^{\prime}, y_{1}^{\prime}, y_{2}^{\prime}\right\} \\
S^{\prime} & =\left\{\left\{x_{1}^{\prime}, y_{1}^{\prime}\right\},\left\{x_{1}^{\prime}, x_{2}^{\prime}, y_{1}^{\prime}, y_{2}^{\prime}\right\}\right\} \\
W^{\prime} & =\left\{\left(x_{1}^{\prime},\left\{x_{1}^{\prime}, y_{1}^{\prime}\right\}\right),\left(x_{1}^{\prime}, X^{\prime}\right),\left(y_{1}^{\prime},\left\{x_{1}^{\prime}, y_{1}^{\prime}\right\}\right),\left(y_{1}^{\prime}, X^{\prime}\right),\left(x_{2}^{\prime}, X^{\prime}\right),\left(y_{2}^{\prime}, X^{\prime}\right)\right\}
\end{aligned}
$$

and the valuation $\theta^{\prime}$ on $\mathcal{F}^{\prime}$ such that $\theta^{\prime}(p)=\left\{x_{1}^{\prime}, x_{2}^{\prime}\right\}, \theta^{\prime}(q)=\left\{x_{1}^{\prime}, y_{1}^{\prime}\right\}$ and $\theta(r)=\emptyset$ for each propositional variable $r \neq p, q$.

It easy to check that $M$ and $M^{\prime}=\left(F^{\prime}, \theta^{\prime}\right)$ are bisimilar in the language without $q$ (bisimilation connects elements without prime and corresponding elements with prime and an index). So $\psi$ is true or false at all bisimilar pairs simultaneously. Formula $\square \hat{K} \diamond K p \rightarrow \diamond K p$ is true in $M$ and after any restriction and hence $M,(x, X) \models \varphi$ and $M,(x, X) \models \psi$ and $M^{\prime},\left(x_{1}^{\prime}, X^{\prime}\right) \models \psi$. But $M^{\prime},\left(x_{1}^{\prime}, X^{\prime}\right) \not \models \psi$ because $M^{\prime},\left(x_{1}^{\prime}, X^{\prime}\right) \not \models[p \vee \neg q](\square \hat{K} \diamond K p \rightarrow \diamond K p)$.

\subsection{Axiomatization and completeness}

The axioms of $w S S L_{a a}$ are all axioms of $w S S L_{a}$ plus the following formulas: $[!] \varphi \rightarrow[\psi] \varphi$ for all formulas $\psi$ in $F_{0 r}$. The rules of inference of $w S S L_{a a}$ are all rules of inference of $w S S L_{a}$ plus the following rule of inference: [!]-special rule (from $\left\{A([\psi] \varphi): \psi\right.$ is a formula in For $\left._{a}\right\}$ infer $A([!] \varphi)$ ). A formula $\varphi$ is said to be $w S S L_{a a}$-provable iff $\varphi$ belongs to the least set of formulas containing all axioms of $w S S L_{a a}$ and closed with respect to all rules of inference of $w S S L_{a a}$. Here, the first result is

Proposition 19. Let $\varphi$ be a formula. If $\varphi$ is $w S S L_{a a}$-provable then $\models \varphi$.

Proof. It suffices to demonstrate the following properties: (i) the axioms of $w S S L_{a a}$ are valid; (ii) the rules of inference of $w S S L_{a a}$ preserve validity. The proof is left to the reader, we only describe the case of the [!]-special rule of inference. Let $A$ be an admissible form and $\varphi$ be a formula such that $\not \models A([!] \varphi)$. Hence, there exists a frame $\mathcal{F}=(X, S, W)$ such that $\mathcal{F} \not \forall A([!] \varphi)$. Thus, there exists a valuation $\theta$ on $\mathcal{F}$ such that $\mathcal{F}, \theta \not \models A([!] \varphi)$. Therefore, there exists $(x, U) \in W$ such that $\mathcal{F}, \theta,(x, U) \not \models A([!] \varphi)$. By induction on $A$, one easily sees that there exists a formula $\psi$ in For $_{a}$ such that $\mathcal{F}, \theta,(x, U) \forall A([\psi] \varphi)$. Consequently, $\mathcal{F}, \theta \not \models A([\psi] \varphi)$. Hence, $\mathcal{F} \not \models A([\psi] \varphi)$. Thus, $\not \models A([\psi] \varphi)$.

Proposition 20. Let $\varphi$ be a formula. If $\models \varphi$ then $\varphi$ is $w S S L_{a a}$-provable. 
Proposition 20 is more difficult to establish than Proposition 19 and we defer proving it till the end of the section. In the meantime, we present some useful results. We shall define $w S S L_{a a}$-theories as sets $\Gamma$ of formulas satisfying the following conditions: (i) $\Gamma$ contains the set of all $w S S L_{a a}$-provable formulas; (ii) $\Gamma$ is closed under the rule of inference of modus ponens; (iii) $\Gamma$ is closed under the [!]-special rule. Of course, the analogue for $w S S L_{a a}$-theories of Proposition 5 holds. See [2, Lemma 4.12] for the proof of a similar result. We shall define the canonical model of $w S S L_{a a}$ in the same way as we have defined the canonical model of $w S S L$. Of course, the analogue for the canonical model of $w S S L_{a a}$ of Lemma 2 holds. Let $\mathcal{M}^{c}=\left(S^{c}, R_{K}^{c}, R_{\square}^{c}, \theta^{c}\right)$ be the canonical model of $w S S L_{a a}$. Considering $\mathcal{M}^{c}$ as a Kripke model where the modal connectives $K$ and $\square$ are interpreted by means of the binary relations $R_{K}^{c}$ and $R_{\square}^{c}$ and where the modal connective [!] is interpreted as in $A P A L$ ( $P A L$ with arbitrary announcements) [2], the proposition below contains a result that can be proved by induction on $\varphi$. See [2, Lemma 4.13] for the proof of a similar result.

Proposition 21. Let $\varphi$ be a formula. For all $\Gamma \in S^{c}$, we have for all finite sequences $\left(\psi_{1}, \ldots, \psi_{n}\right)$ of formulas, $\mathcal{M}^{c}, \Gamma=\left[\psi_{1}\right] \ldots\left[\psi_{n}\right] \varphi$ iff $\left[\psi_{1}\right] \ldots\left[\psi_{n}\right] \varphi \in \Gamma$.

We shall define the unraveling of the canonical model of $w S S L_{a a}$ in the same way as we have defined the unraveling of the canonical model of $w S S L$. Of course, the analogue for the unraveling of the canonical model of $w S S L_{a a}$ of Lemma 3 holds. Let $\Gamma_{0}$ be a maximal consistent $w S S L_{a a}$-theory and $\mathcal{M}^{u}=\left(S^{u}, R_{K}^{u}, R_{\square}^{u}, \theta^{u}\right)$ be the unraveling of $\mathcal{M}^{c}$ around $\Gamma_{0}$. Considering $\mathcal{M}^{u}$ as a Kripke model where the modal connectives $K$ and $\square$ are interpreted by means of the binary relations $R_{K}^{u}$ and $R_{\square}^{u}$ and where the modal connective [!] is interpreted as in APAL [2], the proposition below contains a result that can be proved by induction on $\varphi$.

Proposition 22. Let $\varphi$ be a formula. For all $\left(i_{1}, \Gamma_{1}, \ldots, i_{m}, \Gamma_{m}\right) \in S^{u}$, we have for all finite sequences $\left(\psi_{1}, \ldots, \psi_{n}\right)$ of formulas, $\mathcal{M}^{u},\left(i_{1}, \Gamma_{1}, \ldots, i_{m}, \Gamma_{m}\right)=$ $\left[\psi_{1}\right] \ldots\left[\psi_{n}\right] \varphi$ iff $\mathcal{M}^{c}, \Gamma_{m} \models\left[\psi_{1}\right] \ldots\left[\psi_{n}\right] \varphi$.

Let $\equiv^{u}$ be the symmetric and transitive closure of $R_{\square}^{u}$ and $\preceq^{u}$ be the transitive closure of $R_{K}^{u} \circ R_{\square}^{u}$. Obviously, $\equiv^{u}$ is reflexive, symmetrical and transitive and $\preceq^{u}$ is reflexive and transitive. Let $\boldsymbol{\Gamma} \in S^{u}$. The equivalence class modulo $\equiv^{u}$ with $\overline{\boldsymbol{\Gamma}}$ as its representative is denoted $[\boldsymbol{\Gamma}]_{\equiv u}$. The set of all equivalence classes of $S^{u}$ modulo $\equiv^{u}$ is denoted $S^{u} / \equiv^{u}$. Let $f$ be the function assigning to each $\boldsymbol{\Gamma} \in S^{u}$ the subset $f(\boldsymbol{\Gamma})$ of $S^{u} / \equiv^{u}$ defined by $[\boldsymbol{\Delta}]_{\equiv u} \in f(\boldsymbol{\Gamma})$ iff $\boldsymbol{\Gamma} \preceq^{u} \boldsymbol{\Delta}$. Since $\preceq^{u}$ is reflexive, then $[\boldsymbol{\Gamma}]_{\equiv^{u}} \in f(\boldsymbol{\Gamma})$. We shall spatialize $\mathcal{M}^{u}$ in the same way as we have spatialized the unraveling of the canonical model of $w S S L$. The spatialization of $\mathcal{M}^{u}$ consists of the frame $\mathcal{F}^{s}=\left(X^{s}, S^{s}, W^{s}\right)$ and the valuation $\theta^{s}$ on $\mathcal{F}^{s}$ defined as follows: (i) $X^{s}$ is the set of all equivalence classes of $S^{u}$ modulo $\equiv^{u}$; (ii) $S^{s}$ is the range of $f$; (iii) $W^{s}$ is the set of all pairs $\left([\boldsymbol{\Gamma}]_{\equiv^{u}}, f(\boldsymbol{\Gamma})\right)$ such that $\boldsymbol{\Gamma} \in S^{u}$; (iv) $\theta^{s}$ is the function assigning to each $p \in \operatorname{Var}$ the subset $\theta^{s}(p)$ of $X^{s}$ defined by $[\boldsymbol{\Gamma}]_{\equiv^{u}} \in \theta^{s}(p)$ iff $\boldsymbol{\Gamma} \in \theta^{u}(p)$. The interesting result is the following

Proposition 23. Let $\varphi$ be a formula. For all $\boldsymbol{\Gamma} \in S^{u}$, we have for all finite sequences $\left(\psi_{1}, \ldots, \psi_{n}\right)$ of formulas, $\mathcal{F}^{s}, \theta^{s},\left([\boldsymbol{\Gamma}]_{\equiv^{u}}, f(\boldsymbol{\Gamma})\right) \models\left[\psi_{1}\right] \ldots\left[\psi_{n}\right] \varphi$ iff $\mathcal{M}^{u}, \boldsymbol{\Gamma}=\left[\psi_{1}\right] \ldots\left[\psi_{n}\right] \varphi$. 


\subsection{Decidability/complexity}

As for the membership problem in the set of all valid formulas, we do know whether it is decidable or not. Remark that the membership problem in the set of all valid formulas defined in the Section 4 of [2] was proved to be undecidable by French and van Ditmarsch [9].

\section{$5 \quad$ Variants and open problems}

There are several ways to continue this research. One way is by adding overlap operator or (and) by considering multiple agents, as in the interesting recent [1]. The other way is to try to return to the classical subset spaces, in particular we can ask what formulas can be announced so that the restricted model would still be a classical subset space.

\section{Acknowledgements}

We make a point of thanking the colleagues of the Institut de recherche en informatique de Toulouse who, by the discussions we had with them, contributed to the development of the work we present today. In particular, we want to thank Andreas Herzig for his helpful comments and his useful suggestions. We thank Yì Wáng for his encouragement and observations. We thank the ICLA reviewers for their constructive comments.

\section{References}

1. Yì N. Wáng and Thomas Ågotnes. Subset Space Public Announcement Logic. proceedings of ICLA. To appear 2013.

2. Balbiani, P., Baltag, A., van Ditmarsch, H., Herzig, A., Hoshi, T., de Lima, T. 'Knowable' as 'known after an announcement'. The Review of Symbolic Logic 1 (2008) 305-334.

3. Başkent, C. Topics in Subset Space Logic. Master Thesis of the Universiteit van Amsterdam (2007).

4. Başkent, C. Public Announcement Logic in Geometric Frameworks, Fundamenta Informaticae 114 (2012) 117.

5. Blackburn, P., de Rijke, M., Venema, Y. Modal Logic. Cambridge University Press (2001).

6. Dabrowski, A., Moss, L., Parikh, R. Topological reasoning and the logic of knowledge. Annals of Pure and Applied Logic 78 (1996) 73-110.

7. Van Ditmarsch, H., van der Hoek, W., Kooi, B. Dynamic Epistemic Logic. Springer (2007).

8. Van Ditmarsch, H., Ruan, J., Verbrugge, R. Sum and product in dynamic epistemic logic. Journal of Logic and Computation 18 (2008) 563-588.

9. French, T., Van Ditmarsch, H. Undecidability for arbitrary public announcement logic. Advances in Modal Logic 2008: 23-42 
10. Goldblatt, R. Logics of Time and Computation. Center for the Study of Language and Computation (1992).

11. Halpern, J. The effect of bounding the number of primitive propositions and the depth of nesting on the complexity of modal logic. Artificial Intelligence 65 (1995) 361-372.

12. Heinemann, B. Regarding overlaps in topologic. In AiML, v. 6, Kings College Publications, London, 2006.

13. Heinemann B. Topology and Knowledge of Multiple Agents, in AAI v. 5290, Springer, Heidelberg, pp. 110, 2008.

14. Kurucz, A. Combining modal logics. In Blackburn, P., van Benthem, J., Wolter, F. (editors): Handbook of Modal Logic. Elsevier (2007) 869-924.

15. Lutz, C. Complexity and succinctness of public announcement logic. In AAMAS, (2006) 137-143.

16. Papadimitriou, C. Computational Complexity. Addison-Wesley (1994).

17. Parikh, R., Moss, L., Steinsvold, C. Topology and epistemic logic. In Handbook of Spatial Logics. Springer (2007) 299-341.

18. Plaza, J. Logics of public communications. Synthese 158 (2007) 165-179.

\section{Appendix}

\section{A counterexample to PAL SSL}

In the original text [3] by Can Başkent the definition is inconsistent. In a more recent text (see [4]) this definition has been corrected, but unfortunately other problems remain. In the following text we will try to present the intrinsic difficulties of introducing public announcements to SSL.

Let us consider the following subset space (in classical sense, see [6]) $M=$ $(X, O, V)$, where $X=\{x, y\}, O=P(X)$ - all subsets of $X$, and the valuation is such that $V(p)=\{y\}$. Then consider formula $\varphi=K \neg p \vee \square p$. The list of all neighborhood situations where $\varphi$ is true is following

$$
(\varphi)=\{(x,\{x\}),(y, X),(y,\{y\})\}
$$

So as suggested in [3] to construct the restricted model $M_{\varphi}=\left(X_{\varphi}, O_{\varphi}, V_{\varphi}\right)$ we need to take

$$
X_{\varphi}=(\varphi)_{1}=\{x \mid \exists U((x, U) \in(\varphi))\}, \quad O_{\varphi}=\left\{U \cap X_{\varphi} \mid \exists x((x, U) \in(\varphi))\right\}
$$

In our case

$$
X_{\varphi}=X \quad O_{\varphi}=O \text { and } M_{\varphi}=M .
$$

So the restricted model after the announcement of formula $\varphi$ which is not valid in $M$ is $M_{\varphi}=M$. This is a problem because formula $[\varphi] K \varphi \leftrightarrow(\varphi \rightarrow$ $K[\varphi] \varphi$ which should be an axiom of PAL is not universally true. In particular $(y, X) \not \models[\varphi] K \varphi$ and $(y, X) \vDash K[\varphi] \varphi$.

The problem as we see it is that subset space frame has inner structure similar to product of frames and the set of situations where a formula is true not always preserves this structure. One way to confront this problem is to consider generalized subset spaces. In this paper we explore this way. 